\title{
Empirischer Vergleich der Lebenswirklichkeit von Geflüchteten in Sammelunterkünften und regulären Wohnungen
}

Lutz Eichholz, Annette Spellerberg und Jussi S. Jauhiainen

\section{Einleitung}

In diesem Beitrag beschäftigen wir uns mit der Frage, inwiefern die Wohnsituation einen Effekt auf die Integration und das Wohlbefinden von Geflüchteten hat. Quantitative und qualitative Forschungen zu Geflüchteten beziehen sich vorwiegend auf in Sammelunterkünften lebende Personen. Welche Veränderungen mit dem Erhalt einer eigenen Wohnung einhergehen und welchen Effekt die Wohnsituation auf den Verlauf der Integration hat, ist noch wenig erforscht.

Der klassische Ansatz von Integration ${ }^{1}$ unterscheidet Integration nach vier Dimensionen, die der Kulturation (Wissen um die Gesellschaft), Platzierung (Verankerung auf dem Arbeits-, Bildungs- und Wohnungsmarkt), Interaktion (Einbindung in soziale Netzwerke und auch Partizipation) und Identifikation (subjektive Verortung). In unserer Studie untersuchen wir Aspekte der Integration im Sinne von Wohnverhältnissen und das damit in Zusammenhang stehende Einkommen, die Sprachkenntnisse und soziale Kontakte sowie Einschätzungen zur eigenen Zukunft. Unsere Hypothese lautet, dass die Art der Unterkunft und die damit einhergehende unterschiedliche Lebenswirklichkeit insbesondere in den ersten Jahren in Deutschland einen starken Einfluss auf das Wohlbefinden der Geflüchteten hat.

Als Fallbeispiele wurden die kleine Großstadt Kaiserslautern (Einwohner:innen 2018: 102.738) und die im ländlichen Kusel gelegene Aufnahmeeinrichtung für Asylbegehrende (AfA) ausgewählt. Kaiserslautern hat seit 2015 ungefähr 3.500 Geflüchtete aufgenommen, das ist ein für eine deutsche Großstadt durchschnittlicher Anteil. Dies gewährleistet eine vorsichtige Übertragbarkeit auf andere Städte.

1 Esser, Aspekte der Wanderungssoziologie. 
Nachdem wir im folgenden Teil den Stand der Forschung zu Geflüchteten auf dem Wohnungsmarkt vorgestellt haben, gehen wir in Kapitel 2 auf unser Studiendesign und in Kapitel 3 auf die Ergebnisse der Befragung ein. Im Fazit erörtern wie die Ergebnisse im Hinblick auf die Unterschiede nach Unterbring bzw. Wohnung und machen Vorschläge für die politische und stadtplanerische Gestaltung.

\section{Wohnsituationen von Geflüchteten}

Durch den in vielen Städten angespannten Wohnungsmarkt, gesetzliche Bestimmungen und damit einhergehende Einschränkungen (Wohnsitzauflage, Königsteiner Schlüssel, Stand des Asylverfahren bzw. Status), zumeist mangelnde Sprachkenntnisse sowie rassistische Diskriminierung ist es für Geflüchtete schwer, eine eigene Wohnung zu finden. ${ }^{2}$ Nach der beschwerlichen Flucht wohnen dementsprechend viele Geflüchtete Monate bis Jahre in Erstaufnahmeeinrichtungen der Bundesländer oder in Gemeinschaftsunterkünften (GU) der Kommunen. ${ }^{3}$ Wird, meistens nur mit erheblicher Unterstützung, eine eigene Wohnung gefunden, ist diese häufig kleiner, dichter belegt und in ungünstigeren Lagen als durchschnittliche Wohnungen. ${ }^{4}$ Die Größe und Qualität einer Wohnung ist von entscheidender Bedeutung für die Wohnzufriedenheit und Lebensqualität der Menschen: Überbelegung hemmt die Erholung sowie die Persönlichkeitsentwicklung, speziell bei Kindern. ${ }^{5}$ Der Großteil von Geflüchteten hat den Wunsch, in Städten zu wohnen und setzt diesen Wunsch nach Möglichkeit auch um. ${ }^{6}$ In Städten steigt der Anteil von Menschen ohne deutsche Staatsbürgerschaft und Geflüchteter in Quartieren mit hohem Anteil von

2 Baier/Siegert, Die Wohnsituation Geflüchteter; Gesemann/Roth, Erfolgsfaktoren der Kommunalen Integration von Geflüchteten; Wegschaider/Kösemen, Faktensammlung Diskriminierung; Antidiskriminierungsstelle des Bundes, Diskriminierungsrisiken für Geflüchtete in Deutschland, S. 8; Foroutan et al., Zwischen Lager und Mietvertrag: Wohnunterbringung geflüchteter Frauen in Berlin und Dresden.

3 Tanis, BAMF-Kurzanalyse, S. 3.

4 Eichholz/Spellerberg, Geflüchtete Menschen in unterschiedlichen Siedlungsräumen; Tanis, BAMF-Kurzanlyse.

5 Burghardt/Kürner, Kind und Wohnen.

6 Tanis, BAMF-Kurzanalyse; Brücker et al., Wohnsitzauflagen reduzieren die Chancen auf Arbeitsmarktintegration; Gliemann/Szypulski, Integration von Flüchtlingen, S. 109; Foroutan et al., Zwischen Lager und Mietvertrag: Wohnunterbringung geflüchteter Frauen in Berlin und Dresden S. 17 f.; Mehl, Aufnahme und Integration von Geflüchteten in ländliche Räume, S. $43 \mathrm{f}$. 
Menschen mit geringem Einkommen überproportional. ${ }^{7}$ Dies bedeutet, das Geflüchtete verstärkt von negativen Effekten von Segregation wie Einschränkungen bezüglich des Erwerbs der deutschen Sprache oder Stigmatisierung betroffen sind. Die für Geflüchtete repräsentative Längsschnittstudie des Sozio-ökonomischen Panels (SOEP) gibt weitere Hinweise für die problematische Situation auf dem Wohnungsmarkt. Ihre Wohnungen sind kleiner, liegen häufiger in atypischen Lagen wie Industriegebieten und sind pro Quadratmeter teurer als die der Mehrheitsgesellschaft. ${ }^{8}$

Reguläres Wohnen am Wohnort der Wahl ist für die Integration in den Arbeits- und privaten Wohnungsmarkt vorteilhafter als bspw. die Schaffung von AnkER-Zentren und die Umsetzung der Wohnsitzauflage. ${ }^{9}$ "Gemeinschaftsunterkünfte erschweren die Integration" lautet auch die Überschrift einer Untersuchung der Robert Bosch Stiftung. ${ }^{10}$ Den Sozialraum betreffen dabei vor allem die ungewöhnlichen Wohnlagen, die zusammen mit der Größe der Unterkunft eine Einbindung in die Nachbarschaft verhindern. $23 \%$ der Gemeinschaftsunterkünfte in Deutschland befinden sich in Industriegebieten. Geflüchtete, die in Einzelunterkünften wohnen, sind nur zu einem Prozent in dieser atypischen Lage untergebracht. ${ }^{11}$ Weitere Nachteile von Gemeinschaftsunterkünften sind die fehlende Privatsphäre durch Mehrfachbelegungen der Zimmer und nicht abschließbare Wohneinheiten, was die Hälfte der in Gemeinschaftsunterkünften lebenden Geflüchteten betrifft. ${ }^{12}$ Dies geht einher mit einer objektiv niedrigeren Sicherheit. Menschen mit kulturellen und nationalen Unterschieden leben gezwungenermaßen auf engem Raum zusammen. Konflikte und Spannungen sind die Folgen und gerade besonders schutzbedürftige Personen, wie allein reisende Frauen, sind gefährdet. ${ }^{13}$ Je nach Ausstattung der Unterkunft kommen die fehlende Möglichkeit, selbst zu kochen, unzureichende sanitäre Anlagen, schlechte bauliche Zustände sowie ein Abhängigkeitsverhältnis von Sozialarbeitenden und Wachpersonal hinzu. Neben der geringen Wohnqualität in den schlecht ausgestatteten Unterkünften ist gerade

7 Helbig/Jähnen, Wie brüchig ist die soziale Architektur unserer Städte?

8 Eichholz/Spellerberg, Geflüchtete Menschen in unterschiedlichen Siedlungsräumen, S. 4 f.; Schacht/Metzing, Lebenssituation von Geflüchteten, S. 281.

9 Brücker et al., Wohnsitzauflagen reduzieren die Chancen auf Arbeitsmarktintegration.

10 Robert Bosch Stiftung, Was wir über Flüchtlinge (nicht) wissen, S. 31.

11 Baier/Siegert, Die Wohnsituation Geflüchteter, S. 8.

12 Baier/Siegert, Die Wohnsituation Geflüchteter; BBSR, Integration von Flüchtlingen in den regulären Wohnungsmarkt.

13 Foroutan et al., Zwischen Lager und Mietvertrag: Wohnunterbringung geflüchteter Frauen in Berlin und Dresden. 
dieses Abhängigkeitsverhältnis zu Sozialarbeitenden integrationshemmend, da eigene Problemlösungskompetenzen zurückgehen. ${ }^{14}$

Die integrationshemmende Unterbringung in Sammelunterkünften betrifft eine Vielzahl von Geflüchteten. Im Jahr 2016 lebten 48 \% von ihnen in Gemeinschaftsunterkünften. Der Anteil schwankt dabei von Bundesland zu Bundesland deutlich. In Rheinland-Pfalz und dem Saarland lebten nur $22 \%$ bzw. $21 \%$ der Geflüchteten in Gemeinschaftsunterkünften. In Brandenburg, Hamburg und Berlin wurden mit $70 \%$ bis $83 \%$ die höchsten Werte erreicht. ${ }^{15} \mathrm{Da}$ bei fortschreitender Integration mehr Chancen auf dem Wohnungsmarkt für Geflüchtete vorhanden sind und durch aktuell weniger nachkommende Geflüchtete mehr Kapazitäten genutzt werden können, verringert sich im Laufe des Aufenthalts der Anteil der in Gemeinschaftsunterkünften Lebenden. ${ }^{16}$

Die Reaktion von Nachbar:innen auf Gemeinschaftsunterkünfte wurde von Jürgen Friedrichs in sechs Stadtteilen in NRW erforscht. Die Ergebnisse zeigen, dass Gemeinschaftsunterkünfte in Gebieten mit höherer Bildung und höheren Einkommen stärker akzeptiert werden und mehr Kontakt zu Geflüchteten selbigen Effekt hat. ${ }^{17}$ Die soziale Lage der Quartiersbewohner:innen ist bei der Untersuchung von Wohnsituationen von Geflüchteten entsprechend zu berücksichtigen.

\section{Studiendesign}

Für die Untersuchung der Auswirkungen von unterschiedlichen Wohnformen auf den Verlauf des Ankommens wurde im Sommer 2019 eine quantitative Befragung in unterschiedlichen Ankunftssituationen und Wohnsituationen in Kaiserslautern und Kusel (RLP) durchgeführt. Zusätzlich wurde eine von uns 2018 und 2019 durchgeführte qualitative Befragung zur Interpretation der quantitativen Daten miteinbezogen.

Die semi-strukturierte Befragung von Geflüchteten wurde in regulären Wohnungen und Gemeinschaftsunterkünften (GU) in Kaiserslautern und in der Aufnahmeeinrichtung für Asylbegehrende (AfA) in Kusel von April bis Juni 2019 durchgeführt. Finanziert wurde diese Studie vom Fachgebiet Stadtsoziologie sowie dem Strategic Research Council an der Academy of

14 Robert Bosch Stiftung, Was wir über Flüchtlinge (nicht) wissen, S. 32.

15 Baier/Siegert, Die Wohnsituation Geflüchteter.

16 Tanis, BAMF-Kurzanalyse, S. 3.

17 Friedrichs et al., Die Akzeptanz von Flüchtlingen. 
Finland von Professor Jussi S. Jauhiainen von der Geografie-Sektion der Universität Turku in Finnland geleitet.

Die Gemeinschaftsunterkünfte, in denen die Befragungen stattfanden, sind von ihrer Ausstattung, Lage und Bewohnerschaft sehr unterschiedlich (vgl. Tab. 1). Die Unterbringung in der AfA ist homogener. Dort leben die Bewohner:innen in Zimmern mit bis zu acht Betten, die in einzelnen Häusern oder Etagen nach Herkunftsland, Geschlecht und Familien belegt werden. Familien haben in der Regel ein Zimmer mit sechs Betten für sich alleine. Gemeinschaftlich genutzte Küchen und sanitäre Einrichtungen werden auf jeder Etage vorgehalten.

Da die Ausgabe der Fragebögen größtenteils persönlich stattfand, wurden für die Befragung von in Wohnungen lebenden Geflüchteten Gebiete ausgewählt, in denen möglichst viele räumlich nah beieinander wohnen (vgl. Abb. 1). Die Adressen wurden uns von der Stadtverwaltung Kaiserslautern zur Verfügung gestellt. Bei der Interpretation der Ergebnisse muss daher berücksichtigt werden, dass Personengruppen, die in Straßen mit wenigen Geflüchteten wohnen, unterrepräsentiert sind. Insgesamt haben wir die Befragung in 53 von 292 Straßen, in denen in Kaiserslautern Geflüchtete wohnen, durchgeführt. Etwas mehr als die Hälfte der Menschen aus den Nationen Syrien, Irak, Iran, Nigeria, Afghanistan, Somalia und Eritrea lebten in den ausgewählten Straßen. Geflüchtete, die außerhalb dieser Gebiete in Kaiserslautern wohnen, wurden nicht in unsere Stichprobe einbezogen. Wir haben somit keine repräsentative, zufällige Stichprobenziehung durchgeführt, aber dennoch eine sehr weitgehende Abdeckung bezüglich der regulär und der in Unterkünften wohnenden Personen erreicht.

290 Geflüchtete haben unsere Fragebögen teilweise oder komplett ausgefüllt. 75 von ihnen lebten in der AfA Kusel, 77 in einer der Gemeinschaftsunterkünfte in Kaiserslautern und 138 lebten in regulären Wohnungen in Kaiserslautern. Die Rücklaufquoten in den einzelnen Gebieten schwankten stark und waren abhängig von der Motivation, Zuverlässigkeit und Offenheit der Befragten. Die Rücklaufquote in der AfA lag bei ungefähr 19\%. Im März 2019 waren rund 390 Geflüchtete in den Gemeinschaftsunterkünften von Kaiserslautern registriert, in der oben genannten Zahl sind auch Minderjährige enthalten, die nicht an der Befragung teilgenommen haben. Die Rücklaufquote in den Gemeinschaftsunterkünften lag bei $17 \%$ (ohne Kinder) und bei den regulär Wohnenden bei $13 \%$. 
Tabelle 1: Besuchte Gemeinschaftsunterkünfte (inkl. AfA)

\begin{tabular}{|c|c|c|c|c|}
\hline $\begin{array}{c}\text { Wohnform / } \\
\text { Bewohnende }\end{array}$ & Küche & Bad & Wohndauer & $\begin{array}{c}\text { Entfernung } \\
\text { zur Fußgän- } \\
\text { gerzone }\end{array}$ \\
\hline Doppelzimmer / 200 & Etagenweise & Etagenweise & 1 -2 Jahre & $<1 \mathrm{~km}$ \\
\hline Einzelzimmer / 36 & Etagenweise & Einzel & Längerfristig & $<1 \mathrm{~km}$ \\
\hline $\begin{array}{c}\text { Einzimmer- } \\
\text { wohnungen / 40 }\end{array}$ & Einzel & Einzel & Längerfristig & $<1 \mathrm{~km}$ \\
\hline Einzelzimmer / 40 & Etagenweise & Etagenweise & Längerfristig & $<2 \mathrm{~km}$ \\
\hline Wohnungen / 109 & Einzel & Einzel & Längerfristig & $<1 \mathrm{~km}$ \\
\hline Wohnungen / 20 & Einzel & Einzel & Längerfristig & $<1 \mathrm{~km}$ \\
\hline Sechsbettzimmer / 400 & Etagenweise & Etagenweise & $1-6$ Monate & $<1 \mathrm{~km}$ \\
\hline
\end{tabular}

Quelle: Statistiken der jeweiligen Unterkunftsverwaltung. Begehungen.

\section{Abbildung 1: Untersuchungsgebiete Kaiserslautern}

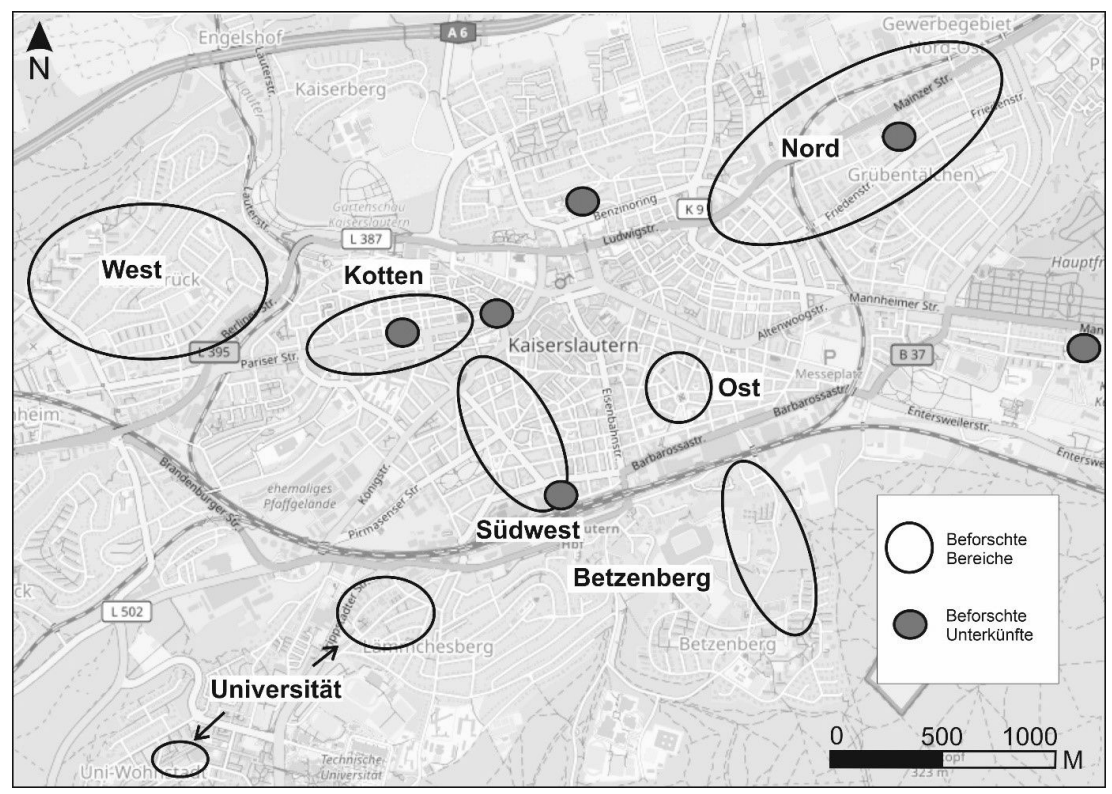

Quelle: Eigene Darstellung auf Basis von Openstreetmaps.

Den Teilnehmenden wurde die Option zur Verfügung gestellt, den Fragebogen in den Sprachen Deutsch, Englisch, Farsi, Arabisch, Somalisch, Tigrinja, Französisch, Türkisch oder Kurdisch auszufüllen. Es wurden allen das Ziel der Untersuchung und der Hinweis auf Anonymität, Vertrau- 
lichkeit der erhobenen Daten und Freiwilligkeit der Teilnahme erläutert. Bei einer Zustimmung gaben wir den Fragebogen aus; die Adressat:innen konnten auch nach Erhalt von der Teilnahme zurücktreten oder einzelne Fragen unbeantwortet lassen. Zudem wurden unausgefüllte Fragebögen zurückgelassen, um auch potentielle Teilnehmende zu erreichen, die den Besuch des Umfrageteams verpasst hatten.

Bei der Interpretation erhobener Daten zu Geflüchteten müssen einige Besonderheiten berücksichtigt werden. Das Antwortverhalten kann verstärkt von sozialer Erwünschtheit geprägt sein und auch die Ausgabe übersetzter Fragebögen kann Verständnisprobleme nicht vollständig verhindern. Gründe für ein von sozialer Erwünschtheit geprägtes Antwortverhalten können vielseitig sein. Misstrauen gegenüber den Interviewer:innen, Befürchtungen, dass die Antworten einen Einfluss auf das eigene Leben in Deutschland haben oder kulturelle Verständnisprobleme sind zentrale Einflussfaktoren, die verstärkt bei Befragungen von Geflüchteten auftreten können. ${ }^{18}$

Als Ergänzung zu der quantitativen Befragung haben wir im Jahr 2018 leitfadengestützte Interviews mit 20 Geflüchteten (neun davon wurden nach 2019 noch einmal befragt), 18 Nachbar:innen und 15 Experten:innen geführt. Ziel der hier als Interpretationshilfe für die Befragung und amtlichen Daten genutzten Interviews war es, die Perspektive der Geflüchteten in verschiedenen Siedlungsräumen in Rheinland-Pfalz zu erheben und den Prozess der Integration zu erfassen. Hierdurch sollten Integrationsmechanismen in Abhängigkeit von der Wohnsituation erforscht werden. Uns interessierte vor allem die Wirkung von unterschiedlichen Wohnlagen, die fußläufige Erreichbarkeit von unterschiedlichen Einrichtungen und sozialen Kontakten sowie die Häufigkeit und Intensität des Austauschs mit Nachbar:innen. ${ }^{19}$

\section{Ergebnisse: Auswirkungen der Wohnverhältnisse auf den Verlauf des Ankommens}

In diesem Teil werden die Ergebnisse der Befragung größtenteils nach Unterkunftsart differenziert dargestellt. Weitere Merkmale (zum Beispiel Auf-

18 Röder et al., Methodische Herausforderungen quantitativer Befragungen von Geflüchteten am Beispiel einer Vorstudie in Sachsen.

19 Für genauere Ergebnisse der Interviews siehe: Eichholz/Spellerberg, Geflüchtete Menschen in unterschiedlichen Siedlungsräumen. 
enthaltsstatus, ländlich oder städtischer Wohnort, Herkunft) sind im englischsprachigen Untersuchungsbericht zu finden. ${ }^{20}$ Die Lebenswirklichkeiten der Geflüchteten unterscheiden sich nach der Art der Unterbringung immens und wurden in diesem Untersuchungsbericht nicht dargelegt.

In der AfA Kusel ist der Alltag stark reguliert - vom Zeitpunkt der Essenausgabe bis zur An- und Abmeldung bei Verlassen der Unterkunft. Es gibt wenig Berührungspunkte mit der Lokalbevölkerung im Alltag. Von medizinischer Hilfe über Deutschunterricht bis zur Sozialberatung werden vielfältige Angebote direkt in der Unterkunft organisiert. In den Gemeinschaftsunterkünften können die Bewohner:innen das Leben eigenständiger als in der AfA organisieren. Auch in dieser Unterbringungsform gibt es Unterstützung direkt in der Unterkunft. Der Unterstützungsumfang variiert stark nach Größe und Art der Unterkunft sowie den dort festgelegten Regeln. Den höchsten Grad an Eigenständigkeit haben die in regulären Wohnungen lebenden Geflüchteten. Formale Aufgaben, wie z.B. der Abschluss eines Mietvertrags oder die Kontrolle der Nebenkostenabrechnung, müssen selbst getätigt werden. Das Umfeld ist deutlich weniger von der Anwesenheit anderer Geflüchteter dominiert. Benötigte Hilfeangebote müssen selbstständig aufgesucht werden.

\subsection{Demographie und Sozialstruktur}

$70 \%$ der Befragten waren männlich und $30 \%$ weiblich (die Antwortmöglichkeit divers wurde nicht abgefragt). Dies stimmt mit der Verteilung von Geflüchteten in Rheinland-Pfalz überein. Insgesamt befanden sich fast alle (97\%) Befragten in der Altersgruppe von 18-49 Jahren. Auch diese Verteilung spiegelt die Demographie aller Geflüchteten in Rheinland-Pfalz und Kaiserslautern wider. ${ }^{21}$

Die Befragten stammten aus 21 Ländern, davon die Hälfte aus Syrien; mit einigem Abstand folgten Personen aus dem Iran (8\%), Nigeria (7\%) und Afghanistan (7\%). Diese Angaben stimmten mit der tatsächlichen Verteilung von Asylsuchenden in Kaiserslautern und der AfA überein. Die Hauptnationen der Geflüchteten in Rheinland-Pfalz wurden angemessen durch die Befragung repräsentiert. ${ }^{22}$ In den Unterkünften variierte die Ver-

20 Jauhiainen/Eichbolz/Spellerberg, Refugees, Asylum Seekers and undocumented Migrants in Germany.

21 Vgl. MFFJIV, Monatliche Zugänge in RLP nach EASY Jahr 2019.

22 Vgl. MFFJIV, Monatliche Zugänge in RLP nach EASY Jahr 2019. 
teilung der Herkunftsländer stark. In der AfA Kusel war die häufigste Nation Nigeria ( $n=19)$, gefolgt von der Türkei ( $n=11)$. In den Gemeinschaftsunterkünften waren es Syrien $(n=24)$ und Afghanistan $(n=13)$. Die in Wohnungen lebenden Geflüchteten stammten in unserer Umfrage am häufigsten aus Syrien $(n=124)$ und mit deutlichem Abstand aus dem Iran $(n=10)$. Die Krisen in den Herkunftsländern spiegelten sich in den unterschiedlichen Zusammensetzungen der Geflüchteten wider. Insbesondere in der AfA variieren die Herkunftsländer der Bewohnenden im Zeitverlauf stark.

Da wir in den qualitativen Interviews die Erfahrung gemacht haben, dass den befragten Geflüchteten ihr genauer Aufenthaltsstatus oft nicht bekannt ist, wurde auf amtliche Termini in der Antwortvorgabe verzichtet. Es wurde stattdessen danach gefragt, wie lange die teilnehmenden Personen in Deutschland bleiben dürfen. Nur wenige der Befragten (6\%) hatten einen unbegrenzten Aufenthaltsstatus, die meisten gaben an, drei Jahre in Deutschland bleiben zu dürfen. 18\% der Befragten gaben an, keinen Aufenthaltsstatus zu haben (vgl. Tab. 2). Die meisten der zuletzt genannten Befragten (32\%) lebten in der AfA Kusel. Es ist anzunehmen, dass diese Teilnehmenden „Dublin-Fälle“ waren, für deren Asylverfahren die Bundesrepublik formal nicht zuständig ist und die potentiell mit einer Aufforderung zur Ausreise und bei Weigerung mit einer anschließenden Abschiebung rechnen müssen ${ }^{23}$.Unter den Befragten waren elf Personen, die ohne Aufenthaltsstatus in Gemeinschaftsunterkünften wohnten. Diese Personen sind mit hoher Wahrscheinlichkeit ausreisepflichtig.

Tabelle 2: Dauer der Aufenthaltserlaubnis, in \%

\begin{tabular}{|c|c|c|c|c|}
\hline Unbegrenzt & 3 Jahre & 1 Jahr & 6 Monate & Ohne \\
\hline 6 & 43 & 13 & 19 & 18 \\
\hline
\end{tabular}

Datenbasis: TU Kaiserslautern/URMI Finnland 2019. $\mathrm{n}=246$.

$37 \%$ der Befragten kamen im Zuge der Fluchtbewegungen im Jahr 2015 nach Rheinland-Pfalz. $22 \%$ der Befragten wohnten erst seit weniger als einem halben Jahr in Deutschland. Nur 4\% der Befragten waren vor 2015 nach Deutschland geflüchtet.

Die Dauer des Aufenthalts in Deutschland nach Unterkunftsart variierte stark (vgl. Tab. 3). Dabei fiel insbesondere auf, dass $63 \%$ der Befragten in den Gemeinschaftsunterkünften seit mehr als 24 Monaten in Deutschland

23 Amtsblatt der Europäischen Union, Verordnung (EU) Nr. 604/2013 des Europäischen Parlaments und des Rates. 
lebten. Gründe hierfür können Schwierigkeiten bei der Wohnungssuche sein und dass einige der Unterkünfte in Kaiserslautern für einen längeren Aufenthalt geplant sind (vgl. Tab. 1). Die Tatsache, dass lediglich $10 \%$ der befragten Personen, die sich seit weniger als zwei Jahren in Deutschland befanden, in regulären Wohnungen lebten, belegt den schwierigen Übergang in den Wohnungsmarkt.

\section{Tabelle 3: Dauer in Deutschland nach Unterkunft, in \%}

\begin{tabular}{|l|c|c|}
\hline Unterkunft & 0-24 Monate & Mehr als 24 Monate \\
\hline Wohnungen & 10 & 90 \\
\hline GU & 37 & 63 \\
\hline AfA & 96 & 4 \\
\hline
\end{tabular}

Datenbasis: TU Kaiserslautern/URMI Finnland 2019. n Wohnungen = 103, n GU = $65, \mathrm{n} \mathrm{AfA}=66$.

\subsection{Soziale Lage: Bildung, Sprachkenntnisse, Erwerbstätigkeit und Einkommen}

Die befragten Geflüchteten verfügten über ein relativ hohes formales Bildungsniveau. $65 \%$ der Befragten gaben an, mindestens eine weiterführende Schule besucht zu haben, und mindestens $41 \%$ hatten eine Universität besucht. Dies war ein höheres Bildungsniveau als das der deutschen Bevölkerung und der Geflüchteten-Stichprobe des SOEP (17\% der Geflüchteten haben einen Universitätsabschluss; Brücker et al. 2019: 54). Die Werte bedeuten jedoch auch, dass wir eine selektierte Stichprobe mit Personen mit höherem Bildungsabschluss analysierten.

Sprachkenntnisse sind eine zentrale Voraussetzung für die gesellschaftliche Teilhabe. Der Selbsteinschätzung nach verfügten $53 \%$ der Befragten über gute oder mittlere Kenntnisse der deutschen Sprache, Frauen jedoch seltener als Männer (11\% im Vergleich zu $18 \%$; nicht tabellarisch ausgewiesen). Von denjenigen, die seit zwei Jahren oder länger in Deutschland lebten, sprachen zwei Drittel Deutsch (gut oder mittel). Bei den Befragten, die weniger als zwei Jahre in Deutschland lebten, waren es lediglich $25 \%$ (vgl. Tab. 4). Da ein Drittel der Befragten auch nach zwei Jahren kaum Deutschkenntnisse aufwiesen, wurde deutlich, dass einige Geflüchtete die angebotenen Sprachkurse nicht wahrnehmen wollten, konnten oder durften oder sie ihnen nicht halfen. Hinderlich könnten eine eingeschränkte Mobilität, gesundheitliche Probleme, fehlende Kinderbetreuung während der Kurse, der Bedarf nach speziellen Kursen (z.B. Deutsch für Analphabeten) oder rechtliche Hindernisse (ohne Status o.ä.) gewesen sein. $78 \%$ der 
in Wohnungen lebenden Personen, $55 \%$ aus den Gemeinschaftsunterkünften und immerhin $16 \%$ aus der AfA ordneten ihre Deutschkenntnisse als gut oder mittel ein.

Tabelle 4: Einschätzung der deutschen Sprachkenntnisse, in \%

\begin{tabular}{|l|c|c|}
\hline Dauer in Deutschland & \multicolumn{2}{|c|}{ Einschätzung der Sprachkenntnisse } \\
\hline & gut / mittel & schlecht / nicht vorhanden \\
\hline $0-23$ Monate & 25 & 75 \\
\hline 24 Monate und mehr & 72 & 28 \\
\hline Insgesamt & 53 & 47 \\
\hline
\end{tabular}

Datenbasis: TU Kaiserslautern/URMI Finnland 2019. $\mathrm{n}=232$.

Die Teilnahme am Erwerbsleben bietet nicht nur die finanzielle Möglichkeit, den eigenen Lebensunterhalt zu verdienen und damit von staatlichen Hilfen unabhängig zu sein, sondern spielt für die Integration eine zentrale Rolle. Für Geflüchtete ist die Aufnahme einer Erwerbstätigkeit jedoch mit deutlich mehr Hürden verbunden als für andere Bevölkerungsgruppen: Ein befristeter Aufenthaltsstatus lässt diese Gruppe von Arbeitnehmerinnen und Arbeitnehmern vergleichsweise unattraktiv erscheinen, vor allem, wenn sie ausgebildet werden müssen oder ihre Ausbildung und Abschlüsse nicht anerkannt werden. Erschwerend kommt hinzu, dass Asylbewerber:innen oder Geduldete eine Erlaubnis von der Ausländerbehörde benötigen. ${ }^{24}$ Sie konkurrieren - mit ihren häufigen sprachlichen Defiziten mit deutschen Staatsbürgern, anderen Menschen ohne deutsche Staatsbürgerschaft und untereinander um Jobs. Zudem sind viele bürokratische Hindernisse bezüglich der Anerkennung von Abschlüssen und des jeweiligen Aufenthaltsstatus zu überwinden. ${ }^{25}$

Zum Zeitpunkt der Befragung gaben $28 \%$ der Teilnehmenden an, erwerbstätig (Vollzeit, Halbzeit oder irregulär) zu sein, $72 \%$ gaben an, nicht erwerbstätig zu sein. Die größten Unterschiede zeigten sich nach Art der Unterkunft (d.h. auch Aufenthaltsdauer in der Bundesrepublik) und Geschlecht der Befragten. Von allen Befragten gingen $38 \%$ der männlichen und nur $8 \%$ der weiblichen Befragten einer Erwerbstätigkeit nach. Gerade bei der Erwerbsarbeit zeigen sich deutliche geschlechtsspezifische Unterschiede, die sowohl auf Geschlechterrollen bei den Zugewanderten, ihre

$24 B A$, Aufenthaltsstatus und Arbeitsmarktzulassung.

25 Salzwedel, Herausforderungen und Chancen der Integration von Geflüchteten in den deutschen Arbeitsmarkt. 
Zuständigkeit für die Kinderbetreuung sowie mangelnder Vorerfahrung zurückzuführen ist. ${ }^{26}$ Dennoch waren die Erwerbsquoten unserer Umfrage etwas höher als in anderen Studien ${ }^{27}$, was auf die längere Aufenthaltsdauer in Deutschland zurückzuführen sein dürfte.

Von den Geflüchteten mit unbefristeter Aufenthaltserlaubnis waren immerhin zwei Drittel beschäftigt, allerdings $20 \%$ in Teilzeit und unregelmäBig. Von den Befragten der AfA gaben $6 \%$ an, Vollzeit und $11 \%$ halbtags zu arbeiten und $6 \%$ illegal beschäftigt zu sein (vgl. Tab. 5). Diese hohen Zahlen für erst kürzlich nach Deutschland Geflüchtete sind vermutlich darauf zurückzuführen, dass ein Viertel der AfA-Bewohnenden am Arbeitsmarktprogramm „Flüchtlingsintegrationsmaßnahmen“ teilnahmen und einige diese geringen Tätigkeiten als Vollzeit oder Halbtags-Arbeit einordneten. In diesem Arbeitsmarktprogramm führen Geflüchtete in Erstaufnahmeeinrichtungen mit 80 Cent pro Stunde bezahlte Hilfstätigkeiten als Dolmetschende, Reinigungskräfte, o.ä. aus. ${ }^{28}$

Tabelle 5: Erwerbsstatus, in \%

\begin{tabular}{|l|c|c|c|c|}
\hline Unterkunft & Vollzeit & Halbtags & illegal Beschäftigt & Erwerbslos \\
\hline Wohnungen & 16 & 11 & 7 & 67 \\
\hline GU & 9 & 9 & 5 & 78 \\
\hline AfA Kusel & 6 & 11 & 6 & 77 \\
\hline
\end{tabular}

Datenbasis: TU Kaiserslautern/URMI Finnland. 2019, $\mathrm{n}$ Wohnungen $=125, \mathrm{GU}=$ $66, \operatorname{AfA}=67$.

Auf die Frage nach der Zufriedenheit mit ihrer Arbeitssituation gaben lediglich $7 \%$ an, unzufrieden zu sein. Die Mehrheit der Befragten war völlig zufrieden (55\%), 38\% waren teilweise zufrieden. Dieses Ergebnis war überraschend, da höchstwahrscheinlich viele der Befragten für ihre Tätigkeiten überqualifiziert waren. Ein Grund könnte sein, dass die Freude überwiegt, überhaupt eine Arbeit auszuüben.

Ein Drittel der in Wohnungen lebenden Befragten war in der Lage, $f_{i-}$ nanzielle Rücklagen zu bilden. Die in der AfA und den Gemeinschaftsunterkünften Wohnenden gaben zu $65 \%$ und $52 \%$ an, etwas Geld zurückle-

26 Brücker et al., Wohnsitzauflagen reduzieren die Chancen auf Arbeitsmarktintegration, S. 49-57; vgl. Jauhiainen/Eichholz/Spellerberg, Refugees, Asylum Seekers and undocumented Migrants in Germany, S. 73.

27 Vgl. Brücker et al., Language skills and employment rate of refugees in Germany improving with time, S. 55 .

28

$B M J V$, Bundesanzeiger. 
gen zu können. Dieser höhere Wert wurde erreicht, weil diese Befragten ihr Einkommen bzw. das Geld, das sie als Aufwandsentschädigung oder Taschengeld erhalten, nicht für Nebenkosten o.ä. ausgeben mussten. Ersparnisse sind für Schutzsuchende besonders wichtig, um Kosten für Schleuser zurückzahlen zu können und die Familie im Herkunftsland zu unterstützen ( $\operatorname{sog}$ „remittances“).

Die Antworten auf die Frage, ob definitiv mehr Geld benötigt wird, um die derzeitige Situation zu verbessern, zeigten ähnliche Ergebnisse wie bei der vorangegangenen Frage. Die Zustimmung zu dieser Frage war sehr hoch bei den in Wohnungen lebenden Geflüchteten (81\%) und noch höher als bei den Befragten aus Unterkünften (68\% und $71 \%$, vgl. Tab. 6). Gründe sind auch hier wieder zum einen die in den Unterkünften vorhandene Grundversorgung, zum anderen die höheren Lebenshaltungskosten in Wohnungen.

\section{Tabelle 6: „Definitiv wird mehr Geld benötigt, um die aktuelle Situation zu verbessern", in \%}

\begin{tabular}{|l|c|c|c|}
\hline Unterkunft & Ja & Ich weiß nicht & Nein \\
\hline Wohnungen & 84 & 10 & 7 \\
\hline GU & 71 & 11 & 18 \\
\hline AfA & 68 & 14 & 18 \\
\hline
\end{tabular}

Datenbasis: TU Kaiserslautern/URMI Finnland 2019. n Wohnungen $=124, \mathrm{n}$ GU = 63, $\mathrm{n}$ AfA $=57$.

\subsection{Wohnverhältnisse, Wohnumgebung und Unterstützung}

Die Wohnstandorte von Geflüchteten werden in erster Linie von Behörden bestimmt. Jeweils mehr als ein Viertel der befragten Geflüchteten wohnte in der AfA in Kusel oder in einer Kaiserslauterer Unterkunft, knapp die Hälfte der Befragten wohnte in regulären Wohnungen. Die Befragten antworteten auf die Frage, warum sie sich entschieden hatten, an ihrem derzeitigen Wohnort in Deutschland zu leben, am häufigsten (47 \%), dass die Zuweisung durch Behörden der Grund für den derzeitigen Wohnort darstellt. Sie nannten an zweiter Stelle familiäre Gründe (16\%). Zwei Drittel der befragten Frauen wohnte in eigenen Wohnungen, während es nur $40 \%$ der Männer waren. Frauen konnten als Interviewpartnerinnen in der AfA jedoch nicht im selben Ausmaß wie Männer gewonnen werden. Sie waren auch seltener in den Gemeinschaftsunterkünften anzutreffen. 
Während in der AfA keine Auffälligkeiten bezüglich des Bildungsgrads zu beobachten waren, so zeigte sich doch, dass Geflüchtete mit geringerem Bildungsgrad überdurchschnittlich häufig in Gemeinschaftsunterkünften lebten (GU: $41 \%$, regulär: $36 \%$ ). Geflüchtete mit höherem Bildungsabschluss waren bei den in Wohnungen lebenden Befragten überrepräsentiert (Gemeinschaftsunterkunft: $21 \%$, Wohnungen: 5 4\%). Die Wohnungssuche ist für Geflüchtete mit befristeten Aufenthaltstiteln, geringem Einkommen, kulturellen Differenzen, niedrigerem Bildungsabschluss und Sprachdefiziten auf dem freien Wohnungsmarkt besonders schwierig. Je nach der Situation auf dem Wohnungsmarkt können sie gezwungen sein, länger in Gemeinschaftsunterkünften zu wohnen, in denen sie sich häufig Küche, Dusche und Toilette teilen müssen. Unzufriedenheit mit den derzeitigen Unterkünften hängt häufig mit der unzureichenden Bereitstellung einer ausreichenden Basisausstattung bezüglich Toiletten oder Duschen zusammen (aber nicht nur damit). Im Allgemeinen waren fast drei von $\operatorname{vier}(71 \%)$ mit dem Angebot völlig zufrieden, und einer von sieben (15\%) gab an, es seien nicht genügend Duschen, Toiletten und andere Einrichtungen vorhanden (vgl. Tab. 7). Männer ( $71 \%$ Zustimmung) und Frauen (69\% Zustimmung) waren dabei fast zu gleichen Anteilen mit den sanitären Einrichtungen zufrieden. Dies war eher eine Ausnahme, da Männer in der Regel zufriedener mit der Unterbringung waren. ${ }^{29}$ Das Ergebnis zeigte, dass die Unterbringung für die Geflüchteten von der Mehrheit der Befragten als zufriedenstellend eingestuft wurde. Dieses Ergebnis kontrastiert mit den unterdurchschnittlichen objektiven Wohnverhältnissen, gemessen an den für unsere Gesellschaft etablierten Standards, wie z.B. Wohnfläche und Ausstattungsmerkmale. Ein möglicher Grund dafür ist, dass die vorherigen Unterkünfte der Befragten, gerade während der Flucht, einen deutlich niedrigeren Standard hatten und dementsprechend die derzeitigen Unterkünfte oder Wohnungen besser bewertet werden.

29 Jauhiainen/Eichholz/Spellerberg, Refugees, Asylum Seekers and undocumented Migrants in Germany, S. $66 \mathrm{f}$. 
Tabelle 7: „In der aktuellen Unterkunft sind genug Toiletten, Duschen, etc. vorhanden", in \%

\begin{tabular}{|l|c|c|c|}
\hline Unterkunft & Stimme zu & Weiß nicht & Stimme nicht zu \\
\hline Wohnungen & 78 & 13 & 9 \\
\hline GU & 65 & 14 & 22 \\
\hline AfA Kusel & 63 & 16 & 21 \\
\hline
\end{tabular}

Datenbasis: TU Kaiserslautern/URMI Finnland. 2019 n Wohnungen = 127, n GU = $65 \mathrm{n}, \mathrm{AfA}=56$.

Der Anteil derjenigen, die sich nicht ausreichend versorgt fühlten, war unter den Geflüchteten der Erstaufnahmeeinrichtung in Kusel und den Gemeinschaftsunterkünften gleich hoch (21\% bzw. $22 \%$ ); die Mehrheit war jedoch auch hier mit der Anzahl an sanitären Einrichtungen zufrieden. Diejenigen, die äußerten, dass nicht genügend sanitäre Einrichtungen zur Verfügung stehen, waren in der Regel auch mit ihrer derzeitigen Stadt oder Nachbarschaft nicht zufrieden und/oder bewerteten ihre Zukunftsperspektiven nicht positiv. Von den in regulären Wohnungen lebenden Geflüchteten war etwa jede:r Zehnte mit der sanitären Ausstattung unzufrieden.

Das Verhältnis zwischen den Angestellten der Unterkunft und den Bewohnenden wurde insgesamt positiv bewertet. Die höchste Zustimmungsrate zu der Frage „In meiner derzeitigen Unterkunft werde ich gut behandelt" gab es unter den Befragten aus den Gemeinschaftsunterkünften (83\%). Dies kann als gutes Zeugnis für die Beschäftigten in diesen Unterkünften gelten. Die länger in der Bundesrepublik und in Wohnungen lebenden Befragten stimmten zu $74 \%$ zu und die eher kurz in der AfA lebenden Befragten zu $70 \%$. Die geringere Zustimmung in der AfA dürften auf ein angespanntes Verhältnis zum Wachpersonal, die Angst vor drohenden Abschiebungen und striktere Zeiteinteilungen, z.B. in Bezug auf Essenszeiten, Öffnungszeiten, Kurse, zurückgehen. Die etwas niedrigeren Zustimmungsraten der in Wohnungen untergebrachten Geflüchteten im Vergleich zu den Bewohner:innen der GU könnte mit Problemen mit Vermieter:innen oder Erfahrungen von Ablehnung durch Nachbar:innen zusammenhängen. Auch wenn Geflüchtete in Wohnungen noch teilweise sozialarbeiterische Unterstützung erhalten, sind sie formal zuständig für die Wohnungen. Daher konnte ein Viertel der Befragten die Frage nicht beantworten, weil sie für regulär Wohnende im Grunde nicht zutrifft (vgl. Tab. 8). Geschlechtsspezifische Unterschiede waren nicht feststellbar. 
Tabelle 8: „In der aktuellen Unterkunft werde ich gut behandelt“, in \%

\begin{tabular}{|l|c|c|c|}
\hline Unterkunft & Stimme zu & Weiß nicht & Stimme nicht zu \\
\hline Wohnungen & 74 & 23 & 3 \\
\hline GU & 83 & 9 & 8 \\
\hline AfA & 70 & 12 & 18 \\
\hline
\end{tabular}

Datenbasis: TU Kaiserslautern/URMI Finnland 2019. $\mathrm{n}$ Wohnungen $=130, \mathrm{n}$ GU $=$ 65, n AfA: 57. Unterschiede sind signifikant (Cramer's V: $\mathrm{p} \leq 0.00$.).

In der AfA war über ein Drittel der Bewohnen:innen mit ihrer Nachbarschaft unzufrieden, in den Gemeinschaftsunterkünften $22 \%$ und in regulären Wohnungen lediglich $12 \%$ (vgl. Tab. 9). In den Sammelunterkünften haben die Bewohner:innen keinen Einfluss auf die Lage und wohnen häufig in einiger Entfernung zu den jeweiligen Zentren und Vierteln, die als „Ankunftsstadtteile“ eingeordnet werden können. ${ }^{30}$

Aus Tabelle 10 ist zu erkennen, dass knapp die Hälfte der in Wohnungen lebenden Geflüchteten mit der Stadt Kaiserslautern zufrieden waren obwohl die Stadt bei Fluchtbeginn nicht bekannt und als Aufenthaltsort gewünscht sein dürfte. Auch die in Unterkünften Untergebrachten waren zu einem hohen Anteil zufrieden, nur jede:r Siebte lehnte die Stadt eindeutig ab.

Tabelle 9: Zufriedenheit mit der Nachbarschaft, in \%

\begin{tabular}{|c|c|c|c|}
\hline Unterkunft & Ja & Ja, teilweise & Nein \\
\hline Wohnungen & 53 & 35 & 12 \\
\hline GU & 41 & 38 & 22 \\
\hline AfA & 31 & 33 & 36 \\
\hline
\end{tabular}

Datenbasis: TU Kaiserslautern/URMI Finnland 2019. n Wohnungen = 131, n GU = 69, n AfA $=55$. Unterschiede sind signifikant (Cramer's V: $\mathrm{p} \leq 0.00$ ).

\section{Tabelle 10: Zufriedenheit mit der Stadt, in \%}

\begin{tabular}{|c|c|c|c|}
\hline Unterkunft & Ja & Ja, teilweise & Nein \\
\hline Wohnungen & 49 & 41 & 10 \\
\hline GU & 46 & 39 & 15 \\
\hline AfA & 30 & 36 & 34 \\
\hline
\end{tabular}

Datenbasis: TU Kaiserslautern/URMI Finnland 2019. $\mathrm{n}$ Wohnungen $=131, \mathrm{n}$ GU = 69, $\mathrm{n}$ AfA $=55$. Unterschiede sind signifikant (Cramer's V: $\mathrm{p} \leq 0.00$ ).

30 Vgl. Spellerberg/Eichholz, Vielfalt leben Kaiserslautern. 
In einer offenen Frage wurden die Befragten gebeten, den präferierten Wohnstandort in Deutschland zu nennen. Bei der Beantwortung dominierten Metropolen wie Frankfurt, Köln, Hamburg, Berlin oder Stuttgart. Kaiserslautern wurde neben diesen Städten am häufigsten (21 Nennungen) genannt. Kusel wurde nicht erwähnt, obwohl 75 der Befragten dort untergebracht waren (vgl. Tab. 11). Dies bestätigt die in der Einleitung dargestellten Erkenntnisse aus anderen Studien, dass Geflüchtete in Großstädten wohnen möchten.

Tabelle 11: Als Wohnstandort bevorzugte Städte, Anzahl Nennungen auf offene Frage

\begin{tabular}{|c|c|}
\hline Kaiserslautern & 21 \\
\hline Frankfurt am Main & 21 \\
\hline Köln & 16 \\
\hline Hamburg & 14 \\
\hline Berlin & 14 \\
\hline München & 10 \\
\hline Stuttgart & 9 \\
\hline
\end{tabular}

Datenbasis: TU Kaiserslautern/URMI Finnland 2019.

\subsection{Soziale Kontakte}

$77 \%$ der Geflüchteten in Wohnungen lebten mit (einem Teil) ihrer Familie zusammen. In den Gemeinschaftsunterkünften und der AfA gaben dies $44 \%$ bzw. $39 \%$ an. Diese Zahlen zeigen, dass viele die Flucht mit zumindest einem Familienmitglied gemeinsam unternahmen oder eine Familienzusammenführung stattgefunden hatte (vgl. Tab. 12). Frauen flohen häufiger im Familienzusammenhang als Männer, denn acht von zehn Frauen, aber nur fünf von zehn Männern lebten mit Familienangehörigen zusammen (vor allem, wenn sie in Wohnungen wohnten, traf diese Aussage auf $85 \%$ der Frauen und $70 \%$ der Männer zu). 
Tabelle 12: „In aktueller Unterkunft bin ich mit einem Teil meiner Familie zusammen", in \%

\begin{tabular}{|c|c|c|c|}
\hline Unterkunft & Stimme zu & Weiß nicht & Stimme nicht zu \\
\hline Wohnungen & 77 & 3 & 20 \\
\hline GU & 44 & 9 & 47 \\
\hline AfA & 39 & 14 & 46 \\
\hline
\end{tabular}

Datenbasis: TU Kaiserslautern/URMI Finnland. 2019. n Wohnungen = 128, n GU = $64, \mathrm{n} \mathrm{AfA}=56$. Unterschiede sind signifikant (Cramer's V: $\mathrm{p} \leq 0.00$.).

Alle Befragten gaben an, Freund:innen aus dem eigenen Land in der Nachbarschaft zu haben. Die in Wohnungen untergebrachten Geflüchteten gaben mit $20 \%$ am häufigsten an, viele Freund:innen aus dem eigenen Land im Nahraum zu haben (vgl. Tab. 11). Dies liegt sicherlich auch daran, dass die Befragung nur in Quartieren durchgeführt wurde, in denen viele Geflüchtete wohnen.

Tabelle 11: Freund:innen aus dem eigenen Land in der Nachbarschaft, in \%

\begin{tabular}{|c|c|c|c|}
\hline Unterkunft & Ja, viele & Ja, einige & Nein \\
\hline Wohnungen & 20 & 80 & 0 \\
\hline GU & 17 & 82 & 0 \\
\hline AfA & 10 & 90 & 0 \\
\hline
\end{tabular}

Datenbasis: TU Kaiserslautern/URMI Finnland 2019. $\mathrm{n}$ Wohnungen $=133, \mathrm{n}$ GU = $70, \mathrm{n}$ AfA $=59$.

Wird allgemein nach Freundschaften mit Deutschen gefragt, so zeigt sich, dass die in Gemeinschaftsunterkünften Wohnenden etwas häufiger als die in Wohnungen lebenden Befragten angaben, mindestens einige deutsche Freund:innen zu haben (69\% in GU, $67 \%$ in Wohnungen, vgl. Tab. 12). Die hohen Anteile von Personen aus Gemeinschaftsunterkünften mit deutschen Freund:innen könnten auch darauf zurückgeführt werden, dass häufig Ehrenamtliche in den Unterkünften tätig sind. In qualitativen Interviews ${ }^{31}$ wurden ehrenamtlich Tätige häufig als deutsche Freund:innen bezeichnet. In der AfA geben nur $26 \%$ an, mindesten einige deutsche Freund:innen zu haben. Dies lässt sich mit dem kurzen Aufenthalt in Deutschland, überwiegend geringen Deutschkenntnissen, und struktureller Exklusion erklären.

31 Eichholz/Spellerberg, Geflüchtete Menschen in unterschiedlichen Siedlungsräumen. 
Tabelle 12: Anzabl deutscher Freund:innen, in \%

\begin{tabular}{|c|c|c|c|}
\hline Unterkunft & Ja, viele & Ja, einige & Nein \\
\hline Wohnungen & 12 & 55 & 34 \\
\hline GU & 15 & 54 & 32 \\
\hline AfA & 6 & 20 & 73 \\
\hline
\end{tabular}

Datenbasis: TU Kaiserslautern/URMI Finnland 2019. n Wohnungen = 134, n GU = 69 , n AfA $=64$. Unterschiede sind signifikant (Cramer's V: $\mathrm{p} \leq 0.00$.).

Je länger und in eigenen Wohnungen die Befragten in Deutschland lebten, umso seltener gaben sie an zu wissen, ob sie von Deutschen freundlich behandelt werden. Knapp $80 \%$ der AfA und GU-Bewohnenden beschrieben Deutsche als freundlich, der Anteil bei den Frauen lag mit $100 \%$ bzw. $91 \%$ noch höher (die Fallzahlen waren mit 14 und 11 allerdings sehr gering). Bei den Geflüchteten in Wohnungen stimmten lediglich zwei Drittel dieser Aussage zu, ein Drittel gab die Antwort „weiß nicht“ (vgl. Tab. 13). Bei dieser Frage kann die vermutete soziale Erwünschtheit einer positiven Antwort das Antwortwortverhalten beeinflusst haben. Geschlechtsspezifische Unterschiede gab es bei in Wohnungen lebenden Befragten nicht. In der SOEP-Befragung deutete sich ähnlich wie bei unseren Ergebnissen an, dass die Sorge vor Rassismus, eine sinkende Willkommenskultur sowie ein Gefühl der Benachteiligung mit steigender Aufenthaltsdauer zunehmen. ${ }^{32}$ Die SOEP-Erhebung ergab, dass knapp die Hälfte der Befragten Erfahrungen mit Diskriminierung in Deutschland hat. ${ }^{33}$ Es kann davon ausgegangen werden, dass die Teilnehmenden unserer Befragung ähnlich häufig Erfahrung mit Diskriminierung machten. Dementsprechend war es bemerkenswert, dass so viele Befragte die Deutschen als freundlich einordneten.

Tabelle 13: „Die Deutschen sind freundlich zu mir“, in \%

\begin{tabular}{|c|c|c|c|}
\hline Unterkunft & Stimme zu & Weiß nicht & Stimme nicht zu \\
\hline Wohnungen & 66 & 33 & 2 \\
\hline GU & 77 & 15 & 8 \\
\hline AfA & 78 & 17 & 4 \\
\hline
\end{tabular}

Datenbasis: TU Kaiserslautern/URMI Finnland 2019. $\mathrm{n}$ Wohnungen $=129, \mathrm{n}$ GU $=$ $65, \mathrm{n}$ AfA $=58$.

32 Lareiro et al., BAMF-Kurzanalyse, S. 11; Scheible et al., Ankommen in Deutschland, S. 45.

33 Brücker et al., IAB-BAMF-SOEP-Befragung von Geflüchteten, S. 64-66. 


\subsection{Zukunftsperspektiven in Deutschland}

Den Teilnehmenden wurden zwei Fragen zum Thema Zukunft im Aufnahmeland gestellt, um herausfinden, was sie vom zukünftigen Leben und dem ihrer Kinder erwarten. Diese Fragen lassen indirekt auch Rückschlüsse auf die aktuelle Lebensqualität zu. Wenn das Leben derzeit sehr unbefriedigend ist, bedeutet schon eine kleine Verbesserung einen Fortschritt. Läuft das Leben in den meisten Bereichen gut, ist es schwerer, es weiter zu verbessern.

Beide Fragen wurden von den in Gemeinschaftsunterkünften oder der AfA Lebenden deutlich positiver beantwortet. 84\% der AfA-Bewohnenden, $74 \%$ der Gemeinschaftsunterkunft-Bewohnenden und $57 \%$ der in Wohnungen Lebenden blickten positiv in die Zukunft (vgl. Tabelle 14, 15). Diese Ergebnisse zeigten, dass Bewohner:innen von Wohnungen pessimistischer eingestellt waren als die Geflüchteten, die in einer Gemeinschaftsunterkunft lebten. Die deutlich pessimistischere Zukunftseinschätzung ist trotzdem bemerkenswert. Obwohl die Befragten in regulären Wohnungen über höhere Erwerbsquoten, mehr Sprachkenntnisse, soziale Kontakte und höhere Zufriedenheiten mit Nachbarschaft sowie mit ihrer Wohnung verfügten, waren sie deutlich pessimistischer. Die längere Aufenthaltsdauer und die Unterbringung in Wohnungen führten teilweise zur Relativierung der positiven Grundstimmung, die noch bei denjenigen mit kürzerer Aufenthaltsdauer und im Schutzraum Lebenden vorherrschte. Ungewissheit ist offensichtlich für viele, die in regulären Wohnungen leben, die passende Antwortkategorie.

\section{Tabelle 14: Wenn ich in Deutschland bleibe, wird mein zukünftiges Leben} besser, in \%

\begin{tabular}{|c|c|c|c|}
\hline Unterkunft & Stimme zu & Weiß nicht & Stimme nicht zu \\
\hline Wohnungen & 57 & 41 & 2 \\
\hline GU & 74 & 24 & 2 \\
\hline AfA & 84 & 15 & 2 \\
\hline
\end{tabular}

Datenbasis: TU Kaiserslautern/URMI Finnland 2019. n Wohnungen = 129, n GU = $66, \mathrm{n}$ AfA $=61$. Unterschiede sind signifikant (Cramer's V: $\mathrm{p} \leq 0.00$.).

Auch die Zukunftsaussichten ihrer Kinder wurden von den schon länger in Deutschland und in regulären Wohnungen lebenden Geflüchteten negativer bewertet. $62 \%$ der in Wohnungen und $85 \%$ der in Gemeinschaftsunterkünften lebenden Befragten gingen von der Möglichkeit aus, dass 
ihre Kinder zukünftig ein gutes Leben in Deutschland haben können (vgl. Tab. 15).

Tabelle 15: „Meine Kinder können zukünftig ein gutes Leben in Deutschland haben", in \%

\begin{tabular}{|c|c|c|c|}
\hline Unterkunft & Stimme zu & Weiß nicht & Stimme nicht zu \\
\hline Wohnungen & 62 & 33 & 5 \\
\hline GU & 85 & 15 & 0 \\
\hline AfA & 82 & 18 & 0 \\
\hline
\end{tabular}

Datenbasis: TU Kaiserslautern/URMI Finnland 2019. n Wohnungen = 129, n GU = $65, \mathrm{n}$ AfA $=62$. Unterschiede sind signifikant (Cramer's V: $\mathrm{p} \leq 0.00$.).

Neben den bereits genannten Gründen kann angenommen werden, dass die optimistischere Einschätzung in Bezug auf ihre Zukunft in Deutschland bei denjenigen mit kürzerer Aufenthaltsdauer auch mit der erst kürzlich überstandenen Flucht zusammenhängt. Im Vergleich zur Flucht dürften sich die Herausforderungen in Deutschland zunächst einfacher darstellen, auch wenn das Asylverfahren als kaum durchschaubar erlebt wird. Erst nach längerem Aufenthalt und mehr Eigenverantwortung außerhalb der Gemeinschaftsunterkünfte treten die Alltagsprobleme und Fremdheit in den Vordergrund. Die Antworten auf die Fragen „Ich würde gerne in mein Ursprungsland zurückkehren“ und „Ich werde wahrscheinlich den Rest meines Lebens in Deutschland verbringen" wiesen ebenfalls in diese Richtung. $5 \%$ der Befragten der AfA, $9 \%$ der in Gemeinschaftsunterkünften und $22 \%$ der in Wohnungen lebenden Geflüchteten wollten in ihr Ursprungsland zurückkehren (vgl. Tab. 16). Gleiches galt für die Einschätzung des Verbleibs in Deutschland. Auch hier waren die Geflüchteten in eigenen Wohnungen deutlich seltener von dieser Möglichkeit überzeugt (vgl. Tab. 17). Zu berücksichtigen ist, dass mit der Aussage „Ich würde gerne in mein Ursprungsland zurückkehren" nur Wünsche und Hoffnungen abseits von konkreten Planungen ausgedrückt werden.

Tabelle 16: "Ich würde gerne in mein Ursprungsland zurückkehren “, in \%

\begin{tabular}{|c|c|c|c|}
\hline Unterkunft & Stimme zu & Weiß nicht & Stimme nicht zu \\
\hline Wohnungen & 22 & 42 & 35 \\
\hline GU & 9 & 40 & 50 \\
\hline AfA & 5 & 21 & 72 \\
\hline
\end{tabular}

Datenbasis: TU Kaiserslautern/URMI Finnland 2019. $\mathrm{n}$ Wohnungen $=127, \mathrm{n}$ GU $=$ $64 \mathrm{n}$ AfA $=57$. Unterschiede sind signifikant (Cramer's V: $\mathrm{p} \leq 0.00$.) 
Tabelle 17: „Ich werde vermutlich den Rest meines Lebens in Deutschland verbringen", in \%

\begin{tabular}{|c|c|c|c|}
\hline Unterkunft & Stimme zu & Weiß nicht & Stimme nicht zu \\
\hline Wohnungen & 46 & 52 & 2 \\
\hline GU & 70 & 24 & 6 \\
\hline AfA & 75 & 20 & 5 \\
\hline
\end{tabular}

Datenbasis: TU Kaiserslautern/URMI Finnland 2019. n Wohnungen = 128, n GU = $66, \mathrm{n}$ AfA $=61$. Unterschiede sind signifikant (Cramer's V: $\mathrm{p} \leq 0.00$.).

Bei der Interpretation unserer Ergebnisse (Tab. 14 bis Tab. 17) ist anzumerken, dass die Unterschiede nach Art der Unterbringung deutlich stärker hervortreten als nach der Aufenthaltsdauer in Deutschland oder dem Aufenthaltsstatus. Daraus lässt sich die Schlussfolgerung ziehen, dass mit dem Bezug einer regulären Wohnung noch deutlicher klar wird, dass das Leben in Deutschland auch längerfristig mit Schwierigkeiten verbunden ist. Die Hypothese, dass die Art des Wohnens und die damit einhergehende unterschiedliche Lebenswirklichkeit einen starken Einfluss auf die Bewertung des Lebens in Deutschland haben, wird somit bestätigt.

\section{Zusammenfassung und Fazit}

Die Ergebnisse des Beitrages zeigen die Einschätzungen und Lebensrealitäten von Geflüchteten insbesondere in Bezug auf ihre Wohnsituation anhand der Fallbeispiele Kaiserslautern und der Aufnahmeeinrichtung für Asylbegehrende in Kusel. Zum Zeitpunkt der Studie war das Alltagsleben der Befragten von Sprachkursen, dem Auseinandersetzen mit der deutschen Bürokratie und dem Hoffen auf eine langfristige Zukunft in Deutschland geprägt. Einige der Befragten hatten begonnen, eine Arbeit aufzunehmen. Die Befragung ergab, dass insbesondere in Wohnungen lebende Geflüchtete keine finanziellen Rücklagen bilden konnten. Die finanziellen Mittel reichten häufig nicht aus, was neben den objektiv geringen Einkommen ${ }^{34}$ auch mit Schulden durch die Flucht oder Rücküberweisungen ins Herkunftsland zusammenhängen kann. Zudem sind einkommensschwache Haushalte von Mietsteigerungen in Städten über-

34 Buntenbach/Jakob/Empen, Geflüchtete auf dem Arbeitsmarkt, S. 1; Brücker et al., Language skills and employment rate of refugees in Germany improving with time, S. 56. 
durchschnittlich betroffen. ${ }^{35}$ Wohnen wollten die meisten Befragten trotz der höheren Kosten in Metropolen oder in Kaiserslautern, nicht aber in Kusel. Zwei Drittel der Bewohner:innen der Gemeinschaftsunterkunft lebten seit mehr als zwei Jahren in Deutschland, also langfristig in Gemeinschaftsunterkünften, in denen wenig Eigenständigkeit möglich ist.

Die Zukunft wird nach dem Umzug in eine reguläre Wohnung seltener positiv eingeschätzt. Obwohl die sprachliche, sozialräumliche und Arbeitsmarktintegration hier weiter vorangeschritten war als bei jenen in den Sammelunterkünften, waren die befragten Geflüchteten hinsichtlich ihrer Zukunft in Deutschland pessimistischer eingestellt. Dass sie keine finanziellen Rücklagen bilden konnten und ein geringerer Teil angab, dass die „Deutschen“ freundlich zu ihnen seien, wird sicherlich einen Einfluss auf diesen Befund gehabt haben.

Die Ergebnisse machten deutlich, dass viele der befragten Geflüchteten in einer Phase des Ankommens waren, die sie vor große Herausforderungen gestellt hat. Nach der beschwerlichen und gefährlichen Flucht und dem durch Warten geprägten Leben in der AfA und in den Gemeinschaftsunterkünften war das eigenständige Leben geprägt von neuen, anfangs nicht leicht zu bewältigenden Anforderungen (Abschluss von Mietverträgen, selbstständiges Bewältigen der Behördengänge, Pflegen von Kontakten außerhalb der Wohnung, Hausarbeit, kulturelle Unterschiede, etc.). Erschwerend kommt hinzu, dass die Problemlösungskompetenzen bei einem langfristigem Aufenthalt in der Gemeinschaftsunterkunft zurückgehen. ${ }^{36}$ Diskriminierungserfahrungen können infolge der aus der Wohnungsunterbringung resultierenden Zunahme von Interaktionen außerhalb der Sammelunterkunft noch einmal zunehmen. Ein weiterer Grund kann schwer verständliche oder räumlich nur schwer erreichbare Hilfe bei länger in Deutschland lebenden Geflüchteten sein. Diese Gruppe benötigt andere Unterstützung als neu Angekommene. ${ }^{37}$ Auf besonders vulnerable Gruppen konnte in diesem Beitrag nicht eingegangen werden (z.B. unbegleitete minderjährige oder stark traumatisierte Geflüchtete).

Die empirischen Ergebnisse sollten zum besseren Verständnis von sozialen und räumlichen Bedingungen für ein zufriedenstellendes Alltagsleben sowie die erfolgreiche Integration in den Wohnungsmarkt und in soziale Netzwerke beitragen. Die zunehmende Perspektivlosigkeit bei steigender

35 Baldenius/Kohl/Schularick, Die neue Wohnungsfrage.

36 Robert Bosch Stiftung, Was wir über Flüchtlinge (nicht) wissen, S. 32.

37 Blank, Wir Schaffen Das!"? Spatial Pitfalls of Neighborhood-Based Refugee Reception in Germany-A Case Study of Frankfurt-Rödelheim. 
Wohndauer und nach dem Umzug in eine reguläre Wohnung ist ein Ergebnis, das zeigt, wie schwierig es für Geflüchtete ist, in Deutschland anzukommen. In den Kommunen sind entsprechend politisch und behördliche Akteur:innen, Zivilgesellschaft, städtische Akteur:innen ebenso wie die Privatwirtschaft gefordert, Integration zu ermöglichen und Abwehrhaltungen abzubauen.

In der Befragung wurde auch die Schwierigkeit von quantitativen Befragungen von vulnerablen Gruppen - wie Geflüchtete es sind - deutlich: Der hohe Anteil der Angabe „weiß nicht“ bei einigen Fragen deutet auf eine große Unsicherheit bei der Darlegung subjektiver Einschätzungen in Befragungssituationen hin. Die sehr positive Bewertung der Unterstützung durch die Mitarbeiter:innen der Gemeinschaftsunterkünfte und der AfA und generell der Unterbringungsbedingungen kann darüber hinaus darauf zurückzuführen sein, dass sich die Befragten mit eigenen, kritischen Wertungen zurückgehalten haben, weil individuelle Konsequenzen befürchtet wurden oder das Antwortverhalten von einer angenommenen sozialen Erwünschtheit geprägt war.

Forschungsbedarf sehen wir bezüglich des Integrationsprozesses in das Erwerbsleben, den Wohnungsmarkt und in soziale Netzwerke, insbesondere außerhalb von Sammelunterkünften. Eine weitere relevante Frage ist, inwiefern die Ernüchterung bei den Befragten, die nach einiger Zeit in Deutschland mit der regulären Wohnunterbringung eintritt, durch mehr ehrenamtlich und amtliche Unterstützung und durch eine kürzere Wohndauer in Gemeinschaftsunterkünften vermindert werden kann. Über den Einfluss der Covid-19-Pandemie auf das Leben in Sammelunterkünften wird vor allem in Bezug auf mögliche Ansteckungsrisiken und eine Verstärkung sozialer Ausschlüsse berichtet. ${ }^{38}$ Wie die in Wohnungen lebenden Geflüchteten die Pandemie kurz- und langfristig bewältigen, ist derzeit noch weitgehend ungeklärt. Die Geflüchteten werden in der PostCorona-Zeit vermutlich zunächst nicht im Zentrum der Aufmerksamkeit stehen. Die Treffpunkte in den Kommunen, die Unterstützungsstrukturen und proaktives Handeln werden wiederzubeleben sein, um die erreichten interkulturellen Interaktionen und Integrationserfolge zu sichern und auszubauen. Inwieweit die sozialen Infrastrukturen erhalten werden können, ist dabei ebenso ungeklärt.

38 Vgl. Schindler, Lockdown in der Flüchtlingsunterkunft; Moskovic, Wie Corona zur Integrationshürde wird; Bozorgmehr et al., SARS-CoV-2 in Aufnahmeeinrichtungen und Gemeinschaftsunterkünften für Geflüchtete. 


\section{Literaturverzeichnis}

Baier, Andreea/Siegert, Manuel: Hrsg. Bundesamt für Migration und Flüchtlinge (BAMF), Die Wohnsituation Geflüchteter, BAMF, Kurzanalyse Ausgabe 02/2018.

Bozorgmehr, Kayvan/Hintermeier, Maren/ Razum, Oliver/Mohsenpour, Amir/ Biddle, Louise/ Oertelt-Prigione, Sabine/Spallek, Jakob/Tallarek, Marie/Jahn, Rosa: SARS-CoV-2 in Aufnahmeeinrichtungen und Gemeinschaftsunterkünften für Geflüchtete, 2020, abrufbar unter: https://pub.uni-bielefeld.de/download /2943665/2943668/FactSheet_PHNetwork-Covid19_Aufnahmeeinrichtungen_v 1_inkl_ANNEX.pdf.

Burghardt, Christa/Kürner, Peter: Kind und Wohnen, Vom Wohnungsgrundriss bis zur Hausordnung, Erfahrungen aus der Praxis, 1994.

Baldenius, Till/Kohl, Sebastian/Schularick, Moritz: Die neue Wohnungsfrage, Gewinner und Verlierer des deutschen Immobilienbooms, in: Leviathan, Jg 48 (2020), Heft 2, 195 - 236.

Blank, Martina: Wir Schaffen Das!"? Spatial Pitfalls of Neighborhood-Based Refugee Reception in Germany-A Case Study of Frankfurt-Rödelheim, 2020.

Brücker, Herbert/Rother, Nina/Schupp, Jürgen: IAB-BAMF-SOEP-Befragung von Geflüchteten: Überblick und erste Ergebnisse. (Forschungsbericht): Hrsg. Bundesamt für Migration und Flüchtlinge (BAMF) Forschungszentrum Migration, Integration und Asyl (FZ), 2016.

Brücker, Herbert/Hauptmann, Johannes/Jaschke, Philipp: Wohnsitzauflagen reduzieren die Chancen auf Arbeitsmarktintegration, IAB-Kurzbericht, 2020.

Bundesagentur für Arbeit (BA): Aufenthaltsstatus und Arbeitsmarktzulassung, 2021, abrufbar unter: https://www.arbeitsagentur.de/unternehmen/arbeitskr aefte/aufenthaltsstatus.

Bundesministerium für Justiz und Verbraucherschutz (BMJV): Bundesanzeiger, Bundesministerium für Arbeit und Soziales, Richtlinie für das Arbeitsprogramm „Flüchtlingsintegrationsmaßnahmen“ vom 20. Juli 2016, Banz AT 27.07.2016 B2.

Buntenbach, Annelie/Jakob, Johannes/Empen, Ruxandra: Geflüchtete auf dem Arbeitsmarkt: Hrsg. DGB Bundesvorstand, arbeitsmarktaktuell Nr. 3 / Juli 2019.

Diskriminierungsrisiken für Geflüchtete in Deutschland: Hrsg. Antidiskriminierungsstelle des Bundes, 2016.

Eichholz, Lutz/Spellerberg, Annette: Geflüchtete Menschen in unterschiedlichen Siedlungsräumen: Hrsg. Burzan, Nicole: Komplexe Dynamiken globaler und lokaler Entwicklungen, Verhandlungen des 39. Kongresses der Deutschen Gesellschaft für Soziologie in Göttingen 2018, 2019.

Esser, Hartmut: Aspekte der Wanderungssoziologie. Assimilation und Integration von Wanderern, ethnischen Gruppen und Minderheiten, Eine handlungstheoretische Analyse, 1980. 
Foroutan, Naika/Hamann, Ulrike/El-Kayed, Nihad/Jorek, Susanna: Zwischen Lager und Mietvertrag: Wohnunterbringung geflüchteter Frauen in Berlin und Dresden, in: Solidarität im Wandel: Berliner Institut für empirische Integrationsund Migrations-forschung (BIM), 2017, 171-195.

Gesemann, Frank/Roth, Roland: Erfolgsfaktoren der Kommunalen Integration von Geflüchteten, 2016.

Gliemann, Katrin/Szypulski, Anja: Integration von Flüchtlingen - Auch eine Frage der Wohnunterbringung, in: Soziale Sicherung im Umbruch: Hrsg. Kaiser, Lutz, 2018, 105-123.

Helbig, Marcel/Jähnen, Stefanie: Wie brüchig ist die soziale Architektur unserer Städte? Trends und Analysen der Segregation in 74 deutschen Städten, Discussion Paper P 2018-001.

Häussermann, Hartmut/Siebel, Walter: Stadtsoziologie - Eine Einführung, 2004.

Integration von Flüchtlingen in den regulären Wohnungsmarkt: Hrsg. Bundesinstitut für Bau., Stadt- und Raumforschung (BBSR), BBSR-Online-Publikation Nr. 21/2017.

Jauhiainen, Jussi/Eichholz, Lutz/Spellerberg, Annette: Refugees, Asylum Seekers and undocumented Migrants in Germany, The Case of Rhineland-Palatinate and Kaiserslautern, 2019.

Jürgen, Friedrichs/Felix, Leßke/Schwarzenberg, Vera: Die Akzeptanz von Flüchtlingen, Eine vergleichende Studie sechs deutscher Wohngebiete, Raumforschung und Raumordnung. Band 77 Heft 4, 2018, 349-366.

Lareiro, Christina de Pavia/Rother, Nina/Siegert, Manuel: BAMF-Kurzanalyse, Ausgabe 01|2020 der Kurzanalysen des Forschungszentrums Migration, Integration und Asyl des Bundesamtes für Migration und Flüchtlinge, Dritte Welle der IABBAMF-SOEP-Befragung von Geflüchteten, Geflüchtete verbessern ihre Deutschkenntnisse und fühlen sich in Deutschland weiterhin willkommen: Hrsg. Bundesamt für Migration und Flüchtlinge (BAMF), 2020.

Mehl, Peter: Aufnahme und Integration von Geflüchteten in ländliche Räume: Spezifika und (Forschungs-) herausforderungen, Beiträge und Ergebnisse eines Workshops am 6. und 7. März 2017 in Braunschweig.

Moskovic, Alexander: Wie Corona zur Integrationshürde wird, tagesschau.de v. 21.11.2020, abrufbar unter: https://www.tagesschau.de/inland/integration-coron a-101.html.

Röder, Atnje/Kailitz, Steffen/Gensweins, Tobias/Rindermann, Sheryn/Schulze Wessel, Julia/Pickel, Gert/Decker, Oliver/Hohenberger, Lea: Methodische Herausforderungen quantitativer Befragungen von Geflüchteten am Beispiel einer Vorstudie in Sachsen, in: Z'Flucht. Zeitschrift für Flüchtlingsforschung 2. Jg. (2018), Heft 2, $313-329$.

Salzwedel, Anja: Herausforderungen und Chancen der Integration von Geflüchteten in den deutschen Arbeitsmarkt, 2018. 
Schacht, Diana/Metzing, Maria: Lebenssituation von Geflüchteten, in: Datenreport 2018, Ein Sozialbericht für die Bundesrepublik Deutschland: Hrsg. Bundeszentrale für politische Bildung (bpb), Statistisches Bundesamt (Destatis), Wissenschaftszentrum für Sozialforschung (Wzb), 2018, S. 280 - 285.

Scheible, Jana/Schacht, Diana/Trübswetter, Parvati: Ankommen in Deutschland: in IAB-Forschungsbericht 13/2017, Aktuelle Ergebnisse aus der Projektarbeit des Instituts für Arbeitsmarkt- und Berufsforschung, IAB-BAMF-SOEP-Befragung von Geflüchteten 2016, Studiendesign, Feldergebnisse sowie Analysen zu schulischer wie beruflicher Qualifikation, Sprachkenntnissen sowie kognitiven Potenzialen, Korrigierte Fassung vom 20. März 2018: Hrsg. Brücker, Herbert/Rother, Nina/Schupp, Jürgen.

Schindler, Franziska: Lockdown in der Flüchtlingsunterkunft, Schulkinder ohne Anschluss, taz Verlags u. Vertriebs GmbH v. 02.02. 2021, abrufbar unter: https:// taz.de/Lockdown-in-der-Fluechtlingsunterkunft/!5748266\&s=Schulkinder+ohne + Anschluss/.

Spellerberg, Annette/Eichholz, Lutz: Vielfalt leben Kaiserslautern, Studie für die Stadt Kaiserslautern, 2018, abrufbar unter: https://kluedo.ub.uni-kl.de/frontdoor /index/index/docId/5686.

Tanis, Kerstin: BAMF-Kurzanalyse. Ausgabe 05|2020 der Kurzanalysen des Forschungszentrums Migration, Integration und Asyl des Bundesamtes für Migration und Flüchtlinge, Entwicklungen in der Wohnsituation Geflüchteter: Hrsg. Bundesamt für Migration und Flüchtlinge (BAMF).

Was wir über Flüchtlinge (nicht) wissen, Eine Expertise im Auf-trag der Robert Bosch Stiftung und des SVR Forschungsbereichs: Hrsg. Robert Bosch Stiftung, 2016.

Wegschaider, Klaudia/Kösemen, Orkan: Hrsg. Bertelsmann Stiftung, Faktensammlung Diskriminierung, Kontext Einwanderungsgesellschaft, 2018. 
